\title{
Therapeutic effects of anti-HMGB1 monoclonal antibody on pilocarpine- induced status epilepticus in mice
}

\author{
Masahiro Nishibori ${ }^{1}$, Li Fu ${ }^{1}$, Hidenori Wake ${ }^{1}$, Dengli Wang ${ }^{1}$, Kiyoshi Teshigawara ${ }^{1}$, Keyue Liu ${ }^{1}$, \\ Hideo K Takahashi ${ }^{2}$, Shuji Mori ${ }^{3}$
}

${ }^{\text {I} P h a r m a c o l o g y, ~ O k a y a m a ~ U n i v e r s i t y ~ G r a d u a t e ~ S c h o o l ~ o f ~ M e d i c i n e, ~ D e n t i s t r y ~ a n d ~ P h a r m a c e u t i c a l ~ S c i e n c e s, ~ J a p a n, ~}$ ${ }^{2}$ Dept of Pharmacology, Faculty of Medicine, Kinki University, Japan, ${ }^{3}$ Dept of Pharmacology, School of Pharmacy, Shujitsu University, Japan

Background: High mobility group box-1 (HMGB1) is a representative damage-associated molecular pattern and has been suggested to be involved in many inflammatory diseases. Among the inhibitors of HMGB1, anti-HMGB1 monoclonal antibody $(\mathrm{mAb})$ might have the strongest neutralizing activity against HMGB1. In the previous studies, we demonstrated the beneficial effects of anti-HMGB1 mAb on brain ischemia- and trauma-induced brain injuries in rats through the inhibition of HMGB1 translocation, protection of BBB and suppression of inflammatory responses. In the present study, we focused on whether anti-HMGB1 antibody treatment could relieve status epilepticus-triggered BBB breakdown and inflammation response in addition to the seizure behavior itself.

Methods: Pilocarpine and methyl-scopolamine were used to establish the acute seizure model. The effects of antiHMGB1 mAb treatment were analyzed with regard to BBB disruption, cytokine expression and behavioral observation. The distribution of therapeutic mAb was also examined immunohistochemically.

Results: Anti-HMGB1 mAb showed inhibitory effects on leakage of the BBB, and on the HMGB1 translocation induced by pilocarpine. The expression of inflammation-related factors, such as MCP-1, CXCL-1, TLR-4, and IL-6 in hippocampus and cerebral cortex were down-regulated by anti-HMGB1 mAb associated with the number of activated astrocytes, microglial cells as well as the expression of IL- $1 \beta$. Both hematoxylin \& eosin and TUNEL staining showed that the apoptotic cells could be reduced after anti-HMGB1 mAb treatment. The onset and latency of Racine stage five were significantly prolonged in the anti-HMGB1 mAb group. In the analysis of localization of therapeutic mAb, we found that the immunoreactivities of i.v. injected anti-HMGB1 mAb were present on/around vascular endothelial cells of brain in pilocarpine-induced seizure mice. The immunostaing of HMGB1 in vascular endothelial cells in pilocarpine-induced seizure mice revealed the release of HMGB1 from vascular endothelial cells.

Conclusion: These results suggested that anti-HMGB1 mAb prevented the BBB permeability, reduced HMGB1 translocation while inhibiting the expression of inflammation-related factors, protected against neural cell apoptosis and prolonged Racine stage 5 seizure onset and latency, leading to the inhibition of status epilepticus and epileptogenesis. 